Letter to the Editor

\title{
Therapeutic management in Sicilian patients with definite arrhythmogenic right ventricular dysplasia/cardiomyopathy and focus on the role of implantable cardioverter-defibrillator therapy
} \author{
Salvatore Novo ${ }^{\mathrm{a}}$, Pasquale Assennato ${ }^{\mathrm{a}}$ \\ a U.O.C. Cardiologia II con Emodinamica, Hospital "P. Giaccone", University of Palermo, Italy \\ ${ }^{\mathrm{b}}$ Heart Failure Unit, ISMETT, Palermo, Italy \\ c Department of Cardiology, ARNAS Ospedale Civico, Palermo, Italy \\ d Department of Cardiology, Maria Eleonora Hospital, Palermo, Italy \\ e Division of Cardiology, Buccheri La Ferla Fatebenefratelli Hospital, Palermo, Italy \\ ${ }^{\mathrm{f}}$ Cardiology Unit, Fondazione Istituto S. Raffaele-G. Giglio, Cefalù, Italy
}

Riccardo M. Inciardi a,*, Antonino Rotolo a , Giuseppe Coppola ${ }^{\text {a }}$, Francesco Clemenza ${ }^{\text {b }}$, Umberto Giordano ${ }^{\text {, }}$, Ernesto Lombardo ${ }^{\mathrm{d}}$, Rossella Schicchi ${ }^{\mathrm{e}}$, Riccardo Torcivia ${ }^{\mathrm{f}}$, Salvatore Arrotti ${ }^{\mathrm{a}}$, Giuseppe Leggio ${ }^{\mathrm{a}}$,

\section{A R T I C L E I N F O}

Article history:

Received 31 December 2013

Accepted 6 January 2014

Available online 23 January 2014

\section{Keywords:}

Cardiomyopathy

Sudden death

Medical management

Implantable cardioverterdefibrillator

Predictors

Arrhythmogenic Right Ventrticular Dysplasia/Cardiomyopathy (ARVD/C) is an inherited cardiomyopathy characterized by right ventricular myocyte loss with fibrofatty replacement, a high risk of ventricular arrhythmias (VA) and sudden cardiac death (SCD) [1]. Prevention of SCD represents the most important management strategy and the achievement of this target can be reached by different therapeutic strategies including implantable cardioverter-defibrillator (ICD) implantation, pharmacologic therapy, catheter ablation of ventricular tachycardia (VT) and cardiac transplantation [2,3]. The aim of this study is to examine the outcome of the different therapies adopted in a group of affected patients, focusing on the role and predictors of ICD therapy.

We conduced a multicenter study evaluating 28 patients (18 male; age $42 \pm 14$ years) with definite ARVD/C. Diagnosis of ARVD/C was based upon the 2010 revised Task Force Criteria [4] and only patients with definite diagnosis entered the study to enhance diagnostic specificity. Management therapy was established according to the clinical

\footnotetext{
* Corresponding author at: U.O.C. Cardiologia II con Emodinamica, Hospital "P. Giaccone", University of Palermo, Via del Vespro 129 Palermo, Italy. Tel.: + 39091343917.

E-mail address: riccardo.inciardi@libero.it (R.M. Inciardi).
}

features and risk stratification of each patient. All patients were followed up at biannual and yearly intervals and data included invasive and noninvasive investigation, and device interrogation. The estimate of the potential survival benefit of ICD was limited to appropriate ICD shock therapies for episode of VF/VFL, since not any arrhythmic events necessarily correspond to the true arrhythmic risk of death. We studied our patients over a mean follow-up of $6.0 \pm 4.4$ years.

Antiarrhythmic drugs were used in 26 patients (93\%), and in 13 patients (50\%) this therapy was associated with ICD implantation [Sotalol in 11 patients (42\%) such as amiodarone; $\beta$-blockers in 4 patient (15\%)]. During the follow-up 8 patients (61\%), that initially received only antiarrhythmic drugs, had an ICD implantation cause of the lack of arrhythmic control. Radiofrequency catheter ablation was performed in 6 patients (21\%) and in no cases its efficacy was observed because of the recurrence of VT that required ICD implantation in whole cases. Cardiac transplantation was performed as a final therapeutic option due to refractory congestive heart failure in 3 patients (10\%).

At the time of the diagnosis 15 patients (54\%) received an ICD implantation based to the estimated risk of SCD, according with the last guidelines [5] for management of patients with VA. During the follow-up 8 patients (61\%) received an ICD because of the relapse of arrhythmic events although they were using antiarrhythmic drugs or underwent catheter ablation. Twenty patients (86\%) had received appropriate ICD therapy. An appropriate ICD shock intervention for ventricular fibrillation (VF)/ventricular flutter (VFL) was seen in 12 patients (52\%). Compared with the 100\% actual survival rate, VF/VFL-free survival rate was $96 \%, 94 \%$ and $51 \%$ respectively at 1,5 and 10 years of follow-up (logrank $\mathrm{p}<0.0001$ ) (Fig. 1). The estimated mortality reduction at $1,5,10$ years of follow-up was $4 \%, 6 \%$ and $49 \%$ and the average rate of ICD interventions for VF/VFL was $5 \%$.

Univariate predictors of ICD shock therapy were a Holter premature ventricular complex (PVC) count $>500 / 24 \mathrm{~h}$ (hazard ratio [HR]: 9.44; 95\% confidence interval [CI]: 1.17 to $15.95 ; \mathrm{p}=0.03$ ) and age presentation $>44$ years ( $\mathrm{HR}: 3.71 ; 95 \% \mathrm{CI}: 1.04$ to $13.19 ; \mathrm{p}=0.04$ ) (Table 1 ). Moreover, both of them remained as significant predictors on multivariable analysis. The positive predictive value (PPV) and the negative 


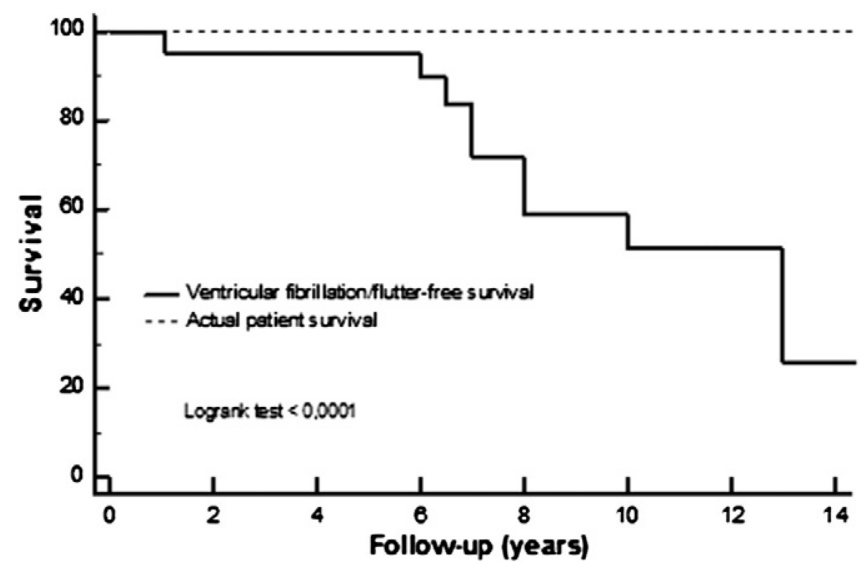

Fig. 1. Kaplan-Meier analysis of survival free of VF/VFL compared with actual patient survival. Divergence between lines reflects the estimated survival benefit of ICD therapy.

predictive value (NPV) of PVCs count $>500 / 24$ h were $88.9 \%$ and $77.7 \%$ respectively, sensitivity and specificity were $80 \%$ and $87.5 \%$ respectively. The cumulative survival rate free of ICD therapy at 10 years was higher (80\%) in patients with low count of PVCs $(\leq 500 / 24 \mathrm{~h})$ compared with patients with a high count of PVCs $(24.5 \%)$ (logrank p $<0.01)$. Also, the cumulative survival rate free of ICD therapy at 10 years was $71 \%$ in patients with age presentation $\leq 44$ years compared with $37 \%$ in patients with age presentation $>44$ years (logrank $\mathrm{p}=0.02$ ). We finally analyzed the relationship between the number of risk factors and ICD shock therapy. We found that patients with one or two risk factors had a significantly increased risk of ICD therapy. The 5-year survival rates free of appropriate ICD shock therapy for patients with 0 or 2 risk factors were $100 \%$ and $75 \%$, respectively (logrank $\mathrm{p}=0.002$ ).

The prevention of life-threatening arrhythmias is the most important management strategy in ARVD/C patients and risk stratification represents an essential step to achieve this target [6]. However in many cases the choice can be a difficult cause of the rarity and the clinical heterogeneity of the disease [7]. According to literature [8-10], even our study confirms the predominant role of ICD implantation as the firstline therapy and suggests the secondary employment of other therapeutic options, when used as unique treatment, only in patients with hemodynamically stable arrhythmias, or asymptomatic mutation carriers or patient with borderline or possible diagnosis of ARVD/C [6]. We moreover identified two clinical variables as significant predictors of appropriate ICD shock therapy and the combination of these risk factors portends an incremental risk. We thought that a very frequent Holter monitoring PVC count represents a marker of electrical instability leading to ventricular arrhythmias. Moreover patients whose presentation starts over the
Table 1

Predictors of appropriate ICD shock intervention.

\begin{tabular}{|c|c|c|c|c|c|c|}
\hline \multirow[t]{2}{*}{ Variable } & \multicolumn{3}{|c|}{ Univariable analysis } & \multicolumn{3}{|c|}{ Multivariable analysis } \\
\hline & \multicolumn{3}{|c|}{ HR 95\%CI p value } & \multicolumn{3}{|c|}{ HR 95\%CI p value } \\
\hline $\begin{array}{l}\text { PVCs }>500 / 24 \text { h on Holter } \\
\text { monitoring }\end{array}$ & 9.44 & $1.17-15.95$ & 0.03 & 9.28 & $1.06-20.55$ & 0.04 \\
\hline Age presentation $>44$ years & 3.71 & $1.04-13.19$ & 0.04 & 6.07 & $1.02-2.5$ & 0.04 \\
\hline NSVT & 2.82 & $0.78-10.10$ & 0.11 & 0.37 & $0.02-4.7$ & 0.45 \\
\hline Syncope & 0.64 & $0.19-2.11$ & 0.47 & & & \\
\hline T wave inversion $\left(V_{1}-V_{3}\right)$ & 0.20 & $0.04-0.91$ & 0.38 & & & \\
\hline Male gender & 0.46 & $0.11-1.96$ & 0.30 & & & \\
\hline Inducibility at EPS & 1.80 & $0.34-9.29$ & 0.48 & & & \\
\hline Epsilon wave & 0.25 & $0.03-2.07$ & 0.20 & & & \\
\hline Prolonged QRS duration & 0.76 & $0.2-2.90$ & 0.69 & & & \\
\hline Biventricular involvement & 1.18 & $0.3-4.56$ & 0.80 & & & \\
\hline Family history of sudden death & 0.65 & $0.19-2.23$ & 0.50 & & & \\
\hline
\end{tabular}

third decade, conceal an important anatomical damage, including myocardial and conduction tissue, underlying their high risk of VA. This finding can be usefully employed to risk-stratify patients who need ICD implantation.

\section{References}

[1] Watkins Hugh, Ashrafian Houman, Redwood Charles. Inherited cardiomyopathies. N Engl J Med April 28 2011;364:1643-56.

[2] Basso Cristina, Corrado Domenico, Marcus Frank I, Nava Andrea, Thiene Gaetano. Arrhythmogenic right ventricular cardiomyopathy. Lancet 2009;373:1289-300.

[3] Calkins H. Arrhythmogenic right ventricular dysplasia. Curr Probl Cardiol 2013 Mar;38(3):103-23.

[4] Marcus FI, McKenna WJ, Sherrill D, et al. Diagnosis of arrhythmogenic right ventricular cardiomyopathy/dysplasia: proposed modification of the task force criteria. Circulation 2010;121:1533-41.

[5] Zipes DP, Camm AJ, Borggrefe M, et al. American College of Cardiology/American Heart Association Task Force; European Society of Cardiology Committee for Practice Guidelines; European Heart Rhythm Association; Heart Rhythm Society. ACC/AHA/ ESC 2006 guidelines for management of patients with ventricular arrhythmias and the prevention of sudden cardiac death: a report of the American College of Cardiology/American Heart Association Task Force and the European Society of Cardiology Committee for Practice Guidelines (Writing Committee to Develop Guidelines for Management of Patients With Ventricular Arrhythmias and the Prevention of Sudden Cardiac Death): developed in collaboration with the European Heart Rhythm Association and the Heart Rhythm Society. Circulation 2006;114:e385-484.

[6] Fernández-Armenta J, Brugada J. Arrhythmogenic right ventricular dyplasia. , 10ESC Council for Cardiology Practice; $2012 \mathrm{~N}^{\circ} 26$.

[7] Wichter T, Paul M, Eckardt L, et al. Arrhythmogenic right ventricular cardiomyopathy. Antiarrhythmic drugs, catheter ablation, or ICD? Herz 2005;30:91-101.

[8] Corrado D, Leoni L, Link MS, et al. Implantable cardioverterdefibrillator therapy for prevention of sudden death in patients with arrhythmogenic right ventricular cardiomyopathy/dysplasia. Circulation 2003;108:3084-91.

[9] Corrado D, Calkins H, Link MS, et al. Prophylactic implantable defibrillator in patients with arrhythmogenic right ventricular cardiomyopathy/dysplasia and no prior ventricular fibrillation or sustained ventricular tachycardia. Circulation 2010;122:1144-52.

[10] Bhonsale A, James CA, Tichnell C, et al. Incidence and predictors of implantable cardioverter defibrillator therapy in patients with arrhythmogenic right ventricular dysplasia/cardiomyopathy undergoing implantable defibrillator implantation for primary prevention. J Am Coll Cardiol 2011;58:1485-96. 